\title{
PTU-061 THE USE OF CAPSULE ENDOSCOPY FOR THE DIAGNOSIS OF SMALL BOWEL TUMOURS: THE FIRST SINGLE CENTRE UK EXPERIENCE
}

doi:10.1136/gut.2011.239301.189

R Sidhu,* M E McAlindon Department of Gastroenterology, Royal Hallamshire Hospital, Sheffield, UK

Introduction Small bowel tumours represent $1-3 \%$ of all gastrointestinal malignancies but can be difficult to diagnose. Since the advent of capsule endoscopy (CE), it has become an important modality in the investigation of patients with small bowel tumours. We conducted a study to evaluate the clinical presentation, endoscopic appearance, diagnostic and therapeutic impact of CE in the management of small bowel tumours.

Methods A retrospective review of consecutive patients that underwent $\mathrm{CE}$ with the findings of small bowel tumours over a seven year period was conducted. Data was collected for patient demographics, indication of CE, findings on CE including location of suspected tumour with subsequent follow up data.

Results 24 patients were found to have a small bowel tumour on CE. This represented $1.5 \%$ of all patients that underwent $\mathrm{CE}$ over seven years. The indications included iron deficiency anaemia $(n=9)$, overt bleeding $(n=11)$ and investigation of complications of coeliac disease $(n=4)$. Patients had a mean number 
of 4.7 tests prior to CE which included gastroscopy, colonoscopy, small bowel radiology, computed tomographic scanning, mesenteric angiograms and red cell labelled scans. The mean age in the group was 55 years (range $34-83$ years). The endoscopic appearances on CE included a mass or ulcerated mass lesion $(n=20)$ or isolated ulceration of mucosa with fresh bleeding $(n=3)$ and multiple ulcers with abnormal underlying mucosa $(n=1)$.

The tumours were located in the jejunum in ten patients, in the ileum in 13 patients and in the fourth part of the duodenum in one patient. The lesions were single in $96 \%$ of cases and multiple in $4 \%(n=1)$. Capsule retention occurred in four patients (16.6\%). 19 patients underwent surgery while two patients were treated with chemotherapy. The tumours were adenocarcinoma $(n=4)$, carcinoid $(n=2)$, gastrointestinal stromal tumour (GIST, $n=5)$, lymphoma $(n=4)$, vascular tumours (haemangioma, angioma, angiomyolipoma, $n=6$ ), metastatic renal cancer $(n=1)$, glomus tumour $(n=1)$ and a benign fibroid tumour $(\mathrm{n}=1)$. Three patients were treated palliatively.

Conclusion CE is an important modality in the diagnostic work up of patients with small bowel tumours and it has a positive impact on patient management. The early use of CE in these patients should be encouraged as it often detects tumours missed by conventional modalities.

Competing interests None.

Keywords capsule endoscopy, small bowel, tumour. 